Bull. Austral. Math. Soc.

$26 \mathrm{~A} 51,90 \mathrm{C} 30$

VoL. $68(2003) \quad[113-125]$

\title{
SUFFICIENT CONDITIONS FOR INVEXITY
}

\author{
NGUYen Xuan Ha and Do Van LuU
}

In this paper, we show that the Robinson, Nguyen-Strodiot-Mifflin and Jourani constraint qualifications are sufficient conditions for invexity of constrained functions with respect to the same scale function in Lipschitzian mathematical programmings. A Kuhn-Tucker necessary optimality condition is also given under the constraint qualification on the invexity property of constrained functions.

\section{INTRODUCTION}

Invexity and its generalisations play an important role in the theory of optimality conditions and duality. The invex concept was firstly introduced by Hanson [6] and Craven [2], and has been extensively studied by many authors (see, for example, [2, $3,4,6,7,8,10,11,14])$. In [2], Craven derived second order sufficient conditions for invexity under the hypotheses on twice differentiability. He showed that the map $f=h \circ g$ is invex at $\bar{x}$ provided that $h$ is invex and $g$ is differentiable with the derivative $g^{\prime}(\bar{x})$ having full rank. Hanson-Rueda [7] established a sufficient condition for invexity by showing the existence of scale functions in nonlinear programming through the use of linear programming. Craven [3] gave a generalisation of the invexity property, not requiring derivatives, which characterises invexity at a point. In general, in nonlinear programming, invexity is sufficient for optimality in conjunction with the Kuhn-Tucker conditions. The invexity of constrained functions yields also the existence of Lagrange multipliers in Kuhn-Tucker necessary conditions for considering problems. A question arises as to when the constrained functions are invex with respect to the same scale function. The purpose of the paper is to answering this question.

In this paper, we establish sufficient conditions for invexity of the constrained functions with respect to the same scale function in Lipschitzian mathematical programmings. Section 2 shows that the regular condition of Robinson type is a sufficient condition for invexity of constrained functions. Sections 3 and 4 are devoted to derivation of another sufficient conditions for invexity. We prove that the Nguyen-StrodiotMifflin and Jourani constraint qualifications are also sufficient conditions for invexity of constrained functions with respect to the same scale function. Finally, a Kuhn-Tucker necessary condition is given under a constraint qualification on the invexity property of constrained functions.

Received 13th January, 2003

This research was partially supported by the Natural Science Council of Vietnam.

Copyright Clearance Centre, Inc. Serial-fee code: 0004-9727/03 \$A2.00+0.00. 


\section{The REgularity CONDITION OF ROBINSON TYPE}

Let $X$ be a Banach space, and let $f, g_{1}, \ldots, g_{m}, h_{1}, \ldots, h_{\ell}$ be real-valued functions defined on $X$. Let $C$ be a nonempty closed subset of $X$. We shall be concerned with the following problem

$$
\left\{\begin{array}{l}
\text { minimize } f(x) \\
\text { subject to } \\
\quad g_{i}(x) \leqslant 0, \quad i=1, \ldots, m, \\
h_{j}(x)=0, \quad j=1, \ldots, \ell, \\
x \in C .
\end{array}\right.
$$

Denote by $M$ the feasible set of Problem (P)

$$
M=\left\{x \in C: g_{i}(x) \leqslant 0, h_{j}(x)=0, i=1, \ldots, m, j=1, \ldots, \ell\right\}
$$

Throughout this paper, we suppose that $f, g_{1}, \ldots, g_{m}, h_{1}, \ldots, h_{\ell}$ are locally Lipschitz at $\bar{x} \in M$. We define the sets $I=\{1, \ldots, m\}, J=\{1, \ldots, \ell\}$, and $I_{0}=\{i \in I$ : $\left.g_{i}(\bar{x})=0\right\}$. Note that the numbers in $I, I_{0}$ and $J$ are ordered from small to large.

Recall ([1]) that the Clarke generalised directional derivative of $f$ at $\bar{x}$, with respect to the direction $d$, is defined as

$$
f^{0}(\bar{x} ; d)=\limsup _{\substack{x \rightarrow \bar{x} \\ t \downarrow 0}} \frac{f(x+t d)-f(x)}{t} .
$$

Following [1], the following set is called the Clarke generalised gradient of $f$ at $\bar{x}$

$$
\partial f(\bar{x})=\left\{\xi \in X^{*}:\langle\xi, d\rangle \leqslant f^{0}(\bar{x} ; d), \forall d \in X\right\}
$$

where $X^{*}$ is the topological dual of $X$. The Clarke tangent cone and the Clarke normal cone to $C$ at $\bar{x}$ are denoted by $T_{C}(\bar{x})$ and $N_{C}(\bar{x})$, respectively,

$$
\begin{aligned}
T_{C}(\bar{x}) & =\left\{v \in X: d_{C}^{0}(\bar{x} ; v)=0\right\} \\
N_{C}(\bar{x}) & =\left\{\xi \in X^{*}:\langle\xi, v\rangle \leqslant 0, \forall v \in T_{C}(\bar{x})\right\}
\end{aligned}
$$

where $d_{C}($.$) is the distance function of C$.

Adapting Reiland's definition ([14]), we shall say that a function $g$ is invex at $\bar{x}$ if there exists a map $\omega$ from $X$ into $T_{C}(\bar{x})$ such that for every $x \in X$,

$$
g(x)-g(\bar{x}) \geqslant g^{0}(\bar{x} ; \omega(x))
$$


Such a map $\omega$ is called a scale.

For the index set $I_{0}$, we denote by $\left|I_{0}\right|$ its cardinal, $\mathbb{R}^{\left|I_{0}\right|}:=\mathbb{R} \times \cdots \times \mathbb{R}\left(\left|I_{0}\right|-\right.$ times), and $\mathbb{R}_{+}^{\left|I_{0}\right|}$ the positive orthant of $\mathbb{R}^{\left|I_{0}\right|}$. Note that $\left|I_{0}\right| \leqslant m$. Denote by $G^{I_{0}}$ the vector-valued function its components are $g_{i}\left(i \in I_{0}\right): G^{I_{0}}(x)=\left(g_{i}(x)\right)_{i \in I_{0}}$. We set

$$
\begin{aligned}
G_{0}^{I_{0}} & =\left(g_{i}^{0}(\bar{x} ; .)\right)_{i \in I_{0}} \\
H & =\left(h_{1}, \ldots, h_{\ell}\right), \quad H_{0}(.)=\left(h_{1}^{0}(\bar{x} ; .), \ldots, h_{\ell}^{0}(\bar{x} ; .)\right) \\
F & =\left(G^{I_{0}}, H\right), \quad F_{0}(.)=\left(G_{0}^{I_{0}}(.), H_{0}(.)\right)
\end{aligned}
$$

The following theorem shows that the regularity condition of Robinson type [16] is a sufficient condition ensuring the constrained functions of Problem (P) to be invex with respect to the same scale.

THEOREM 2.1. Assume that

$$
0 \in \operatorname{int}\left[F_{0}\left(T_{C}(\bar{x})\right)+\mathbb{R}_{+}^{\left|I_{0}\right|} \times\left\{0_{\ell}\right\}\right]
$$

where $0_{\ell}$ is the origin of $\mathbb{R}^{\ell}$. Then the functions $g_{i}\left(i \in I_{0}\right)$ and $h_{j}(j \in I)$ have the following invexity property: there exists a map $\omega: X \rightarrow T_{C}(\bar{x})$ such that for every $x \in X$,

$$
\begin{aligned}
& g_{i}(x)-g_{i}(\bar{x}) \geqslant g_{i}^{0}(\bar{x} ; \omega(x)) \quad\left(\forall i \in I_{0}\right), \\
& h_{j}(x)-h_{j}(\bar{x})=h_{j}^{0}(\bar{x} ; \omega(x)) \quad(\forall j \in J) .
\end{aligned}
$$

Proof: Since $F_{0}($.$) is positively homogeneous, T_{C}(\bar{x})$ and $\mathbb{R}_{+}^{\left|I_{0}\right|} \times\left\{0_{\ell}\right\}$ are cones, it follows that $F_{0}\left(T_{C}(\bar{x})\right)+\mathbb{R}_{+}^{\left|I_{0}\right|} \times\left\{0_{\ell}\right\}$ is a cone. We invoke the regularity condition (1) to deduce that

$$
F_{0}\left(T_{C}(\bar{x})\right)+\mathbb{R}_{+}^{\left|I_{0}\right|} \times\left\{0_{\ell}\right\}=\mathbb{R}^{\left|I_{0}\right|+\ell}
$$

For any $x \in X$, is follows from (2) that

$$
F(x)-F(\bar{x}) \in F_{0}\left(T_{C}(\bar{x})\right)+\mathbb{R}_{+}^{\left|I_{0}\right|} \times\left\{0_{\ell}\right\} .
$$

Hence, there exists $d \in T_{C}(\bar{x})$ such that

$$
F(x)-F(\bar{x}) \in F_{0}(d)+\mathbb{R}_{+}^{\left|I_{0}\right|} \times\left\{0_{\ell}\right\} .
$$

Putting $\omega(x)=d$, we obtain

$$
F(x)-F(\bar{x})-F_{0}(\omega(x)) \in \mathbb{R}_{+}^{\left|I_{0}\right|} \times\left\{0_{\ell}\right\}
$$


Consequently, for every $x \in X$,

$$
\begin{aligned}
g_{i}(x)-g_{i}(\bar{x}) \geqslant g_{i}^{0}(\bar{x} ; \omega(x)) & \left(i \in I_{0}\right), \\
h_{j}(x)-h_{j}(\bar{x})=h_{j}^{0}(\bar{x} ; \omega(x)) & (j \in J) .
\end{aligned}
$$

CoRollary 2.1. If condition (1) is replaced by condition (2), then the conclusion of Theorem 2.1 still holds.

Proof: It is easy to see that (2) implies (1). Moreover, from the proof of Theorem 2.1 we can see that (1) implies (2). Hence, (1) is equivalent to (2), as was to be shown. $\square$

ThEOREM 2.2. Assume that

$$
0 \in G_{0}^{I_{0}}\left(T_{C}(\bar{x})\right)+\mathbb{R}_{++}^{\left|I_{0}\right|}
$$

where $\mathbb{R}_{++}^{\left|I_{0}\right|}=\left\{\left(r_{i}\right)_{i \in I_{0}} \in \mathbb{R}^{\left|I_{0}\right|}: r_{i}>0, \forall i \in I_{0}\right\}$. Then the functions $g_{i}\left(i \in I_{0}\right)$ are invex at $\bar{x}$ with respect to the same scale, that is, there exists a map $\omega: X \rightarrow T_{C}(\bar{x})$ such that for all $x \in X$,

$$
g_{i}(x)-g_{i}(\bar{x}) \geqslant g_{i}^{0}(\bar{x} ; \omega(x)) \quad\left(\forall i \in I_{0}\right)
$$

PRoOF: It is obvious that $G_{0}^{I_{0}}\left(T_{C}(\bar{x})\right)+\mathbb{R}_{++}^{\left|I_{0}\right|}$ is an open subset of $\mathbb{R}^{\left|I_{0}\right|}$. Moreover,

$$
G_{0}^{I_{0}}\left(T_{C}(\bar{x})\right)+\mathbb{R}_{++}^{\left|I_{0}\right|} \subset G_{0}^{I_{0}}\left(T_{C}(\bar{x})\right)+\mathbb{R}_{+}^{\left|I_{0}\right|}
$$

So, it follows readily from (3) that

$$
0 \in \operatorname{int}\left[G_{0}^{I_{0}}\left(T_{C}(\bar{x})\right)+\mathbb{R}_{+}^{\left|I_{0}\right|}\right]
$$

By an argument analogous to that used for the proof of Theorem 2.1 we deduce that there exists a map $\omega: X \rightarrow T_{C}(\bar{x})$ such that for all $x \in X$,

$$
g_{i}(x)-g_{i}(\bar{x}) \geqslant g_{i}^{0}(\bar{x} ; \omega(x)) \quad\left(\forall i \in I_{0}\right)
$$

\section{The NguYen-Strodiot-Mifflin CONSTRAint QUALIFICATION}

It is well known that the Mangasarian-Fromovitz constraint qualification ([12]) has been extended by Nguyen-Strodiot-Mifflin [13] to the case where inequality constraints are Lipschitz. In this section we shall shows that the Nguyen-Strodiot-Mifflin constraint qualification is a sufficient condition for invexity of the constraint functions with respect to the same scale function. 
TheOREM 3.1. Assume that $h_{1}, \ldots, h_{\ell}$ are Fréchet differentiable at $\bar{x}$ with Fréchet derivatives $\nabla h_{1}(\bar{x}), \ldots, \nabla h_{\ell}(\bar{x})$. Suppose also that there exists $d_{0} \in \operatorname{int} T_{C}(\bar{x})$ such that

(i) $\left\langle\nabla h_{j}(\bar{x}), d_{0}\right\rangle=0 \quad(\forall j \in J)$;

(ii) $\left\langle\xi_{i}, d_{0}\right\rangle<0 \quad\left(\forall \xi_{i} \in \partial g_{i}(\bar{x}), \forall i \in I_{0}\right)$;

(iii) $\nabla h_{1}(\bar{x}), \ldots, \nabla h_{\ell}(\bar{x})$ are linearly independent.

Then the functions $g_{i}\left(i \in I_{0}\right)$ and $h_{j}(j \in J)$ have the following invexity property: there exists a map $\omega: X \rightarrow T_{C}(\bar{x})$ such that for every $x \in X$,

$$
\begin{aligned}
g_{i}(x)-g_{i}(\bar{x}) & \geqslant g_{i}^{0}(\bar{x} ; \omega(x)) \quad\left(\forall i \in I_{0}\right), \\
h_{j}(x)-h_{j}(\bar{x}) & =\left\langle\nabla h_{j}(\bar{x}), \omega(x)\right\rangle \quad(\forall j \in J) .
\end{aligned}
$$

Proof: In view of the weak* compactness of $\partial g_{i}(\bar{x})\left(i \in I_{0}\right)$, it follows from assumption (ii) that

$$
g_{i}^{0}\left(\bar{x} ; d_{0}\right)=\max _{\xi_{i} \in \partial g_{i}(\bar{x})}\left\langle\xi_{i}, d_{0}\right\rangle<0 \quad\left(\forall i \in I_{0}\right),
$$

which together with assumption (i) implies that for all $\mu \in \mathbb{R}_{+}^{\left|I_{0}\right|} \backslash\{0\}$ and $\nu \in \mathbb{R}^{\ell}$,

$$
\sum_{i \in I_{0}} \mu_{i} g_{i}^{0}\left(\bar{x} ; d_{0}\right)+\sum_{j \in J} \nu_{j}\left\langle\nabla h_{j}(\bar{x}), d_{0}\right\rangle<0,
$$

where $\mu=\left(\mu_{i}\right)_{i \in I_{0}}, \nu=\left(\nu_{j}\right)_{j \in J}$.

Setting

$$
F_{1}(.)=\left(\left(g_{i}^{0}(\bar{x} ; .)\right)_{i \in I_{0}}, \quad\left(\left\langle\nabla h_{j}(\bar{x}), .\right\rangle\right)_{j \in J}\right),
$$

we shall prove that

$$
F_{1}\left(T_{C}(\bar{x})\right)+\mathbb{R}_{+}^{\left|I_{0}\right|} \times\left\{0_{\ell}\right\}=\mathbb{R}^{\left|I_{0}\right|+\ell} .
$$

Assume the contrary, that

$$
F_{1}\left(T_{C}(\bar{x})\right)+\mathbb{R}_{+}^{\left|I_{0}\right|} \times\left\{0_{\ell}\right\} \underset{\neq}{\subset \mathbb{R}^{\left|I_{0}\right|+\ell}} .
$$

Then, there exists $u=\left(u_{1}, u_{2}\right) \in \mathbb{R}^{\left|I_{0}\right|} \times \mathbb{R}^{\ell}, u \notin F_{1}\left(T_{C}(\bar{x})\right)+\mathbb{R}_{+}^{\left|I_{0}\right|} \times\left\{0_{\ell}\right\}$. Since $F_{1}($.$) is \mathbb{R}_{+}^{\left|I_{0}\right|} \times\left\{0_{\ell}\right\}$-convex, $T_{C}(\bar{x})$ and $\mathbb{R}_{+}^{\left|I_{0}\right|} \times\left\{0_{\ell}\right\}$ are convex cones, it follows that $F_{1}\left(T_{C}(\bar{x})\right)+\mathbb{R}_{+}^{\left|I_{0}\right|} \times\left\{0_{\ell}\right\}$ is a nonempty convex cone in $\mathbb{R}^{\left|I_{0}\right|+\ell}$.

Making use of a separation theorem of disjoint convex sets in finite dimensional space (see, for example, [15, Theorem 11.3]), we claim that there exists $\left(\mu^{*}, \nu^{*}\right)$ $\in \mathbb{R}^{\left|I_{0}\right|} \times \mathbb{R}^{\ell} \backslash\{0\}$ such that

$$
\left\langle\mu^{*}, u_{1}\right\rangle+\left\langle\nu^{*}, u_{2}\right\rangle \leqslant\left\langle\mu^{*}, y\right\rangle+\left\langle\nu^{*}, z\right\rangle\left(\forall(y, z) \in A:=F_{1}\left(T_{C}(\bar{x})\right)+\mathbb{R}_{+}^{\left|I_{0}\right|} \times\left\{0_{\ell}\right\}\right) .
$$


Since $A$ is a convex cone, by virtue of $[5$, Lemma 5.1$]$, we deduce that

$$
\left\langle\mu^{*}, y\right\rangle+\left\langle\nu^{*}, z\right\rangle \geqslant 0 \quad(\forall(y, z) \in A)
$$

which implies that

$$
\begin{array}{r}
\sum_{i \in I_{0}} \mu_{i}^{*} g_{i}^{0}(\bar{x} ; d)+\sum_{j \in J} \nu_{j}^{*}\left\langle\nabla h_{j}(\bar{x}), d\right\rangle \geqslant 0 \quad\left(\forall d \in T_{C}(\bar{x})\right), \\
\left\langle\mu^{*}, z\right\rangle \geqslant 0 \quad\left(\forall z \in \mathbb{R}_{+}^{\left|I_{0}\right|}\right),
\end{array}
$$

where $\mu^{*}=\left(\mu_{i}^{*}\right)_{i \in I_{0}}, \nu^{*}=\left(\nu_{j}^{*}\right)_{j \in J}$.

Since $\left(\mathbb{R}_{+}^{\left|I_{0}\right|}\right)^{*}=\mathbb{R}_{+}^{\left|I_{0}\right|}$, by (6) it follows that $\mu^{*} \in \mathbb{R}_{+}^{\left|I_{0}\right|}$. We shall show that $\mu^{*} \neq 0$.

If this were not so, that is $\mu^{*}=0$, from (5) there would be

$$
\sum_{j \in J} \nu_{j}^{*}\left\langle\nabla h_{j}(\bar{x}), d\right\rangle \geqslant 0 \quad\left(\forall d \in T_{C}(\bar{x})\right)
$$

Since $d_{0} \in \operatorname{int} T_{C}(\bar{x})$, there exists $\delta>0$ such that $B\left(d_{0} ; \delta\right) \subset T_{C}(\bar{x})$, where $B\left(d_{0} ; \delta\right)$ stands for the open ball of radius $\delta$ around $d_{0}$. Hence,

$$
\sum_{j \in J} \nu_{j}^{*}\left\langle\nabla h_{j}(\bar{x}), d\right\rangle \geqslant 0 \quad\left(\forall d \in B\left(d_{0} ; \delta\right)\right)
$$

For any $0 \neq d \in X$, since $B\left(d_{0} ; \delta\right)-d_{0}$ is an open ball of radius $\delta$ centred at 0 , it follows that $t d \in B\left(d_{0} ; \delta\right)-d_{0} \quad(\forall t \in(0, \delta / d))$, which means that $d_{0}+t d \in B\left(d_{0} ; \delta\right)$ $(\forall t \in(0, \delta / d))$. Consequently, in view of (7) and assumption (i), we deduce that for all $t \in(0, \delta / d)$,

$$
\sum_{j \in J} \nu_{j}^{*}\left\langle\nabla h_{j}(\bar{x}), d_{0}+t d\right\rangle=\sum_{j \in J} \nu_{j}^{*}\left\langle\nabla h_{j}(\bar{x}), t d\right\rangle \geqslant 0
$$

whence

$$
\sum_{j \in J} \nu_{j}^{*}\left\langle\nabla h_{j}(\bar{x}), d\right\rangle \geqslant 0 \quad(\forall 0 \neq d \in X)
$$

The above inequality holds trivially if $d=0$. Hence,

$$
\sum_{j \in J} \nu_{j}^{*} \nabla h_{j}(\bar{x})=0
$$


which yields that $\nu_{j}^{*}=0(\forall j \in J)$, since the system $\left\{\nabla h_{j}(\bar{x}), j \in J\right\}$ is linearly independent. That is $\nu^{*}=0$, which conflicts with $\left(\mu^{*}, \nu^{*}\right) \neq 0$. Therefore, $\mu^{*} \neq 0$. Thus $\mu^{*} \in \mathbb{R}_{+}^{\left|I_{0}\right|} \backslash\{0\}, \nu^{*} \in \mathbb{R}^{\ell}$ and

$$
\sum_{i \in I_{0}} \mu_{i}^{*} g_{i}^{0}(\bar{x} ; d)+\sum_{j \in J} \nu_{j}^{*}\left\langle\nabla h_{j}(\bar{x}), d\right\rangle \geqslant 0 \quad\left(\forall d \in T_{C}(\bar{x})\right)
$$

But this contradicts (4). Hence,

$$
F_{1}\left(T_{C}(\bar{x})\right)+\mathbb{R}_{+}^{\left|I_{0}\right|} \times\left\{0_{\ell}\right\}=\mathbb{R}^{\left|I_{0}\right|+\ell} .
$$

For $x \in X$, it follows from (8) that

$$
F(x)-F(\bar{x}) \in F_{1}\left(T_{C}(\bar{x})\right)+\mathbb{R}_{+}^{\left|I_{0}\right|} \times\left\{0_{\ell}\right\}
$$

which implies that there exists $z \in T_{C}(\bar{x})$ such that

$$
F(x)-F(\bar{x}) \in F_{1}(z)+\mathbb{R}_{+}^{\left|I_{0}\right|} \times\left\{0_{\ell}\right\} .
$$

Defining the map $\omega: x \longmapsto \omega(x)=z$, we obtain

$$
F(x)-F(\bar{x})-F_{1}(\omega(x)) \in \mathbb{R}_{+}^{\left|I_{0}\right|} \times\left\{0_{\ell}\right\}
$$

which means that

$$
\begin{aligned}
g_{i}(x)-g_{i}(\bar{x}) & \geqslant g_{i}^{0}(\bar{x} ; \omega(x)) \quad\left(\forall i \in I_{0}\right), \\
h_{j}(x)-h_{j}(\bar{x}) & =\left\langle\nabla h_{j}(\bar{x}), \omega(x)\right\rangle \quad(\forall j \in J) .
\end{aligned}
$$

The proof is complete.

By an argument similar to that used for the proof of Theorem 3.1, we obtain the following

ThEOREM 3.2. Assume that $h_{j}=0 \quad(\forall j \in J)$ and there exists $d_{0} \in T_{C}(\bar{x})$ such that

$$
g_{i}^{0}\left(\bar{x} ; d_{0}\right)<0 \quad\left(\forall i \in I_{0}\right) .
$$

Then, there exists a map $\omega: X \rightarrow T_{C}(\bar{x})$ such that $g_{i}\left(\forall i \in I_{0}\right)$ are invex at $\bar{x}$ with respect to the same scale $\omega$, that is,

$$
g_{i}(x)-g_{i}(\bar{x}) \geqslant g_{i}^{0}(\bar{x} ; \omega(x)) \quad\left(\forall x \in X, \forall i \in I_{0}\right) .
$$

REMARK 3.1. In Theorem $3.2, T_{C}(\bar{x})$ is not necessary to have nonempty interior. 


\section{The Jourani constraint QUalification}

In this section, we show that the constraint qualification of Jourani type ([9]) is a sufficient condition ensuring that all the functions $g_{i}\left(i \in I_{0}\right)$ and $h_{j}(j \in J)$ are invex respect to the same scale. It should be noted here that $h_{j}(j \in J)$ are not necessarily Fréchet differentiable.

THEOREM 4.1. Assume that for all $(\mu, \nu) \in \mathbb{R}_{+}^{\left|I_{0}\right|} \times \mathbb{R}^{\ell} \backslash\{0\}$,

$$
0 \notin \sum_{i \in I_{0}} \mu_{i} \partial g_{i}(\bar{x})+\sum_{j \in J} \nu_{j} \partial h_{j}(\bar{x})+N_{C}(\bar{x}),
$$

where $\mu=\left(\mu_{i}\right)_{i \in I_{0}}, \nu=\left(\nu_{j}\right)_{j \in J}$. Then the functions $g_{i}\left(i \in I_{0}\right)$ and $h_{j}(j \in J)$ have the following invexity property: there exists a map $\omega: X \rightarrow T_{C}(\bar{x})$ such that for every $x \in X$,

$$
\begin{aligned}
g_{i}(x)-g_{i}(\bar{x}) & \geqslant g_{i}^{0}(\bar{x} ; \omega(x)) & & \left(\forall i \in I_{0}\right), \\
h_{j}(x)-h_{j}(\bar{x}) & =h_{j}^{0}(\bar{x} ; \omega(x)) & & (\forall j \in J) .
\end{aligned}
$$

Proof: Since $\partial g_{i}(\bar{x})\left(i \in I_{0}\right)$ and $\partial h_{j}(\bar{x})(j \in J)$ are weak ${ }^{*}$ compact convex in $X^{*}$ and the cone $N_{C}(\bar{x})$ is weak* closed, it follows that for each $(\mu, \nu) \in \mathbb{R}_{+}^{\left|I_{0}\right|} \times \mathbb{R}^{\ell} \backslash\{0\}$, the set

$$
B:=\sum_{i \in I_{0}} \mu_{i} \partial g_{i}(\bar{x})+\sum_{j \in J} \nu_{j} \partial h_{j}(\bar{x})+N_{C}(\bar{x})
$$

is weak* closed convex. On using (9), we obtain $0 \notin B$. Applying a separation theorem (see, for example, [5, Theorem 3.6]) yields that there exists $0 \neq d_{0} \in X$ such that

$$
\left\langle\xi, d_{0}\right\rangle<0 \quad(\forall \xi \in B),
$$

which implies that

$$
\left\langle\xi, d_{0}\right\rangle<0 \quad\left(\forall \xi \in \sum_{i \in I_{0}} \mu_{i} \partial g_{i}(\bar{x})+\sum_{j \in J} \nu_{j} \partial h_{j}(\bar{x})\right) .
$$

Hence,

$$
\begin{aligned}
\sum_{i \in I_{0}} \mu_{i}\left\langle\xi_{i}, d_{0}\right\rangle+\sum_{j \in J} \nu_{j}\left\langle\eta_{j}, d_{0}\right\rangle<0 & \\
& \left(\forall \xi_{i} \in \partial g_{i}(\bar{x}), \forall \eta_{j} \in \partial h_{j}(\bar{x}), \forall i \in I_{0}, \forall j \in J\right) .
\end{aligned}
$$

In view of the weak* compactness of $\partial g_{i}(\bar{x})\left(i \in I_{0}\right)$ and $\partial h_{j}(\bar{x})(j \in J)$, it follows from (11) that

$$
\sum_{i \in I_{0}} \mu_{i} \max _{\xi_{i} \in \partial g_{i}(\bar{x})}\left\langle\xi_{i}, d_{0}\right\rangle+\sum_{j \in J} \nu_{j} \max _{\eta_{j} \in \partial h_{j}(\bar{x})}\left\langle\eta_{j}, d_{0}\right\rangle<0
$$


which leads to the following

$$
\sum_{i \in I_{0}} \mu_{i} g_{i}^{0}\left(\bar{x} ; d_{0}\right)+\sum_{j \in J} \nu_{j} h_{j}^{0}\left(\bar{x} ; d_{0}\right)<0
$$

Next we have to show that $d_{0} \in T_{C}(\bar{x})$. Suppose the contrary, that $d_{0} \notin T_{C}(\bar{x})$. Then, there exists $\zeta \in N_{C}(\bar{x})$ such that

$$
\left\langle\zeta, d_{0}\right\rangle>0
$$

Hence, for $\tilde{\xi}_{i} \in \partial g_{i}(\bar{x})\left(i \in I_{0}\right), \tilde{\eta}_{j} \in \partial h_{j}(\bar{x})(j \in J)$ and $t>0$ large enough, we have

$$
\sum_{i \in I_{0}} \mu_{i}\left\langle\tilde{\xi}_{i}, d_{0}\right\rangle+\sum_{j \in J} \nu_{j}\left\langle\widetilde{\eta}_{j}, d_{0}\right\rangle+t\left\langle\zeta, d_{0}\right\rangle \geqslant 0
$$

So, observing $\sum_{i \in I_{0}} \mu_{i} \tilde{\xi}_{i}+\sum_{j \in J} \nu_{j} \tilde{\eta}_{j}+t \zeta \in B$, we can see that (13) conflicts with (10). Consequently, $d_{0} \in T_{C}(\bar{x})$.

We now prove that

$$
F_{0}\left(T_{C}(\bar{x})\right)+\mathbb{R}_{+}^{\left|I_{0}\right|} \times\left\{0_{\ell}\right\}=\mathbb{R}^{\left|I_{0}\right|+\ell}
$$

Assume the contrary, that

$$
F_{0}\left(T_{C}(\bar{x})\right)+\mathbb{R}_{+}^{\left|I_{0}\right|} \times\left\{0_{\ell}\right\} \underset{\mathbb{R}^{\left|I_{0}\right|+\ell}}{\subsetneq}
$$

Then, there exists

$$
u=\left(u_{1}, u_{2}\right) \in \mathbb{R}^{\left|I_{0}\right|} \times \mathbb{R}^{\ell} \backslash\left[F_{0}\left(T_{C}(\bar{x})\right)+\mathbb{R}_{+}^{\left|I_{0}\right|} \times\left\{0_{\ell}\right\}\right]
$$

Since $F_{0}($.$) is \mathbb{R}_{+}^{\left|I_{0}\right|+\ell}$-convex, it follows that $F_{0}\left(T_{C}(\bar{x})\right)+\mathbb{R}_{+}^{\left|I_{0}\right|} \times\left\{0_{\ell}\right\}$ is convex. Observing $F_{0}($.$) is positively homogeneous, one gets F_{0}\left(T_{C}(\bar{x})\right)+\mathbb{R}_{+}^{\left|I_{0}\right|} \times\left\{0_{\ell}\right\}$ is a nonempty convex cone in $\mathbb{R}^{\left|I_{0}\right|+\ell}$.

Applying a separation theorem for the disjoint convex sets $\{u\}$ and $F_{0}\left(T_{C}(\bar{x})\right)$ $+\mathbb{R}_{+}^{\left|I_{0}\right|} \times\left\{0_{\ell}\right\}$ in the finite dimensional space $\mathbb{R}^{\left|I_{0}\right|+\ell}$ (see, for example, [15, Theorem 11.3]) yields the existence of $\left(\mu^{*}, \nu^{*}\right) \in \mathbb{R}^{\left|I_{0}\right|} \times \mathbb{R}^{\ell} \backslash\{0\}$ such that

$$
\left\langle\mu^{*}, u_{1}\right\rangle+\left\langle\nu^{*}, u_{2}\right\rangle \leqslant\left\langle\mu^{*}, y\right\rangle+\left\langle\nu^{*}, z\right\rangle\left(\forall(y, z) \in F_{0}\left(T_{C}(\bar{x})\right)+\mathbb{R}_{+}^{\left|I_{0}\right|} \times\left\{0_{\ell}\right\}\right) .
$$

Since $F_{0}\left(T_{C}(\bar{x})\right)+\mathbb{R}_{+}^{\left|I_{0}\right|} \times\left\{0_{\ell}\right\}$ is a cone, taking account of [5, Lemma 5.1] we get

$$
\left\langle\mu^{*}, u_{1}\right\rangle+\left\langle\nu^{*}, u_{2}\right\rangle \leqslant 0 \leqslant\left\langle\mu^{*}, y\right\rangle+\left\langle\nu^{*}, z\right\rangle\left(\forall(y, z) \in F_{0}\left(T_{C}(\bar{x})\right)+\mathbb{R}_{+}^{\left|I_{0}\right|} \times\left\{0_{\ell}\right\}\right)
$$


which implies that

$$
\begin{aligned}
\left\langle\mu^{*}, G_{0}^{I_{0}}(d)\right\rangle+\left\langle\nu^{*}, H_{0}(d)\right\rangle \geqslant 0 & & \left(\forall d \in T_{C}(\bar{x})\right), \\
\left\langle\mu^{*}, y\right\rangle \geqslant 0 & & \left(\forall y \in \mathbb{R}_{+}^{\left|I_{0}\right|}\right) .
\end{aligned}
$$

It follows readily from $(16)$ that $\mu^{*} \in\left(\mathbb{R}_{+}^{\left|I_{0}\right|}\right)^{*}=\mathbb{R}_{+}^{\left|I_{0}\right|}$. Hence,

$$
\left(\mu^{*}, \nu^{*}\right) \in \mathbb{R}_{+}^{\left|I_{0}\right|} \times \mathbb{R}^{\ell} \backslash\{0\} .
$$

Moreover, by (15) one gets

$$
\sum_{i \in I_{0}} \mu_{i}^{*} g_{i}^{0}(\bar{x} ; d)+\sum_{j \in J} \nu_{j}^{*} h_{j}^{0}(\bar{x} ; d) \geqslant 0 \quad\left(\forall d \in T_{C}(\bar{x})\right)
$$

which contradicts (12). Consequently, (14) holds.

Taking $x \in X$, it follows from (14) that

$$
F(x)-F(\bar{x}) \in F_{0}\left(T_{C}(\bar{x})\right)+\mathbb{R}_{+}^{\left|I_{0}\right|} \times\left\{0_{\ell}\right\}
$$

which implies that there exists $d \in T_{C}(\bar{x})$ such that

$$
F(x)-F(\bar{x}) \in F_{0}(d)+\mathbb{R}_{+}^{\left|I_{0}\right|} \times\left\{0_{\ell}\right\}
$$

Defining a map $\omega: x \longmapsto \omega(x)=d$, we obtain

$$
F(x)-F(\bar{x})-F_{0}(\omega(x)) \in \mathbb{R}_{+}^{\left|I_{0}\right|} \times\left\{0_{\ell}\right\}
$$

which leads to the following

$$
\begin{aligned}
g_{i}(x)-g_{i}(\bar{x}) \geqslant g_{i}^{0}(\bar{x} ; \omega(x)) & \left(\forall i \in I_{0}\right), \\
h_{j}(x)-h_{j}(\bar{x})=h_{j}^{0}(\bar{x} ; \omega(x)) & (\forall j \in J) .
\end{aligned}
$$

This concludes the proof.

\section{KUHN-TUCKER NECESSARY CONDITIONS}

Let us introduce the following constraint qualification

Condition 5.1.

(i) The function $g_{i}\left(i \in I_{0}\right)$ and $h_{j}(j \in J)$ have the invexity property at $\bar{x}$, that is, there exists a map $\omega: X \rightarrow T_{C}(\bar{x})$ such that for all $x \in X$, 


$$
\begin{aligned}
g_{i}(x)-g_{i}(\bar{x}) \geqslant g_{i}^{0}(\bar{x} ; \omega(x)) & \left(\forall i \in I_{0}\right), \\
h_{j}(x)-h_{j}(\bar{x})=h_{j}^{0}(\bar{x} ; \omega(x)) & (\forall j \in J) ;
\end{aligned}
$$

(ii) for every $(\mu, \nu) \in \mathbb{R}_{+}^{\left|I_{0}\right|} \times \mathbb{R}^{\ell} \backslash\{0\}$, there is $x \in X$ such that

$$
\left\langle\mu, G^{I_{0}}(x)\right\rangle+\langle\nu, H(x)\rangle<0 .
$$

A Kuhn-Tucker necessary optimality condition for Problem (P) can be stated as follows.

Theorem 5.1. Let $\bar{x}$ be a local minimiser of Problem $(P)$. Then, there exist $\bar{\lambda} \geqslant 0, \bar{\mu}_{i} \geqslant 0(i \in I)$ and $\bar{\nu}_{j}(j \in J)$, not all zero, such that for all $d \in T_{C}(\bar{x})$,

$$
\begin{gathered}
\bar{\lambda} f^{0}(\bar{x} ; d)+\sum_{i \in I_{0}} \bar{\mu}_{i} g_{i}^{0}(\bar{x} ; d)+\sum_{j \in J} \bar{\nu}_{j} h_{j}^{0}(\bar{x} ; d) \geqslant 0 \\
\bar{\mu}_{i} g_{i}(\bar{x})=0 \quad(\forall i \in I) .
\end{gathered}
$$

Moreover, if Condition 5.1 is satisfied, then $\bar{\lambda}>0$, it can be taken $\bar{\lambda}=1$.

ProOF: According to [1, Theorem 6.1.1] there exists $\bar{\lambda} \geqslant 0, \bar{\mu}_{i} \geqslant 0(i \in I)$ and $\bar{\nu}_{j} \in \mathbb{R}(j \in J)$, not all zero, such that

$$
\begin{gathered}
0 \in \bar{\lambda} \partial f(\bar{x})+\sum_{i \in I} \bar{\mu}_{i} \partial g_{i}(\bar{x})+\sum_{j \in J} \bar{\nu}_{j} \partial h_{j}(\bar{x})+N_{C}(\bar{x}) \\
\sum_{i \in I} \bar{\mu}_{i} g_{i}(\bar{x})=0 .
\end{gathered}
$$

Since $\bar{\mu}_{i} g_{i}(\bar{x}) \leqslant 0(\forall i \in I)$, it follows from (20) that

$$
\bar{\mu}_{i} g_{i}(\bar{x})=0 \quad(\forall i \in I) .
$$

For $i \in I \backslash I_{0}, g_{i}(\bar{x})<0$, and hence $\bar{\mu}_{i}=0$. Consequently, in view of (19), we get

$$
0 \in \bar{\lambda} \partial f(\bar{x})+\sum_{i \in I_{0}} \bar{\mu}_{i} \partial g_{i}(\bar{x})+\sum_{j \in J} \bar{\nu}_{j} \partial h_{j}(\bar{x})+N_{C}(\bar{x}),
$$

which implies that for every $d \in T_{C}(\bar{x})$,

(21) $\bar{\lambda} \max _{\xi \in \partial f(\bar{x})}\langle\xi, d\rangle+\sum_{i \in I_{0}} \bar{\mu}_{i} \max _{\zeta_{i} \in \partial g_{i}(\bar{x})}\left\langle\zeta_{i}, d\right\rangle$

$$
+\sum_{j \in J} \bar{\nu}_{j} \max _{\eta_{j} \in \partial h_{j}(\bar{x})}\left\langle\eta_{j}, d\right\rangle+\sup _{\gamma \in N_{C}(\bar{x})}\langle\gamma, d\rangle \geqslant 0
$$


Observing that $\sup _{\gamma \in N_{C}(\bar{x})}\langle\gamma, d\rangle \leqslant 0$, from (21) we obtain

$$
\bar{\lambda} \max _{\xi \in \partial f(\bar{x})}\langle\xi, d\rangle+\sum_{i \in I_{0}} \bar{\mu}_{i} \max _{\zeta_{i} \in \partial g_{i}(\bar{x})}\left\langle\zeta_{i}, d\right\rangle+\sum_{j \in J} \bar{\nu}_{j} \max _{\eta_{j} \in \partial h_{j}(\bar{x})}\left\langle\eta_{j}, d\right\rangle \geqslant 0
$$

which leads to (17).

We now assume that condition 5.1 holds, and we should show that $\bar{\lambda}>0$.

Suppose the contrary, that $\bar{\lambda}=0$. Then, by (17) we get

$$
\sum_{i \in I_{0}} \bar{\mu}_{i} g_{i}^{0}(\bar{x} ; d)+\sum_{j \in J} \bar{\nu}_{j} h_{j}^{0}(\bar{x} ; d) \geqslant 0 \quad\left(\forall d \in T_{C}(\bar{x})\right) .
$$

We set $\bar{\mu}=\left(\bar{\mu}_{i}\right)_{i \in I}, \bar{\mu}_{I_{0}}=\left(\bar{\mu}_{i}\right)_{i \in I_{0}}$ and $\bar{\nu}=\left(\bar{\nu}_{j}\right)_{j \in J}$. Since $(\bar{\lambda}, \bar{\mu}, \bar{\nu}) \neq 0$, it follows that $\left(\bar{\lambda}, \bar{\mu}_{I_{0}}, \bar{\nu}\right) \neq 0$, and hence $\left(\bar{\mu}_{I_{0}}, \bar{\nu}\right) \neq 0$. By virtue of condition 5.1 (ii), there exists $\widehat{x} \in X$ such that

$$
\left\langle\bar{\mu}_{I_{0}}, G^{I_{0}}(\widehat{x})\right\rangle+\langle\bar{\nu}, H(\widehat{x})\rangle<0 .
$$

We invoke condition 5.1 (i) to deduce that for all $x \in X$,

$$
F(x)-F(\bar{x})-F_{0}(\omega(x)) \in \mathbb{R}_{+}^{\left|I_{0}\right|} \times\left\{0_{\ell}\right\}
$$

where

$$
\begin{aligned}
F & =\left(G^{I_{0}}, H\right)=\left(\left(g_{i}\right)_{i \in I_{0}},\left(h_{j}\right)_{j \in J}\right), \\
F_{0}(.) & =\left(G_{0}^{I_{0}}(.), H_{0}(.)\right)=\left(\left(g_{i}^{0}(\bar{x} ; .)\right)_{i \in I_{0}},\left(h_{j}^{0}(\bar{x} ; .)\right)_{j \in J}\right) .
\end{aligned}
$$

This leads to the following

$$
F(X) \subset F_{0}\left(T_{C}(\bar{x})\right)+\mathbb{R}_{+}^{\left|I_{0}\right|} \times\left\{0_{\ell}\right\},
$$

as $F(\bar{x})=0$. Hence, there are $\widehat{d} \in T_{C}(\bar{x})$ and $\widehat{v} \in \mathbb{R}_{+}^{\left|I_{0}\right|}$ such that

$$
F(\widehat{x})=F_{0}(\widehat{d})+(\widehat{v}, 0) .
$$

Combining (23) and (24) yields that

$$
\left\langle\bar{\mu}_{I_{0}}, G^{I_{0}}(\widehat{x})\right\rangle+\langle\bar{\nu}, H(\widehat{x})\rangle=\left\langle\bar{\mu}_{I_{0}}, G_{0}^{I_{0}}(\widehat{d})\right\rangle+\left\langle\bar{\nu}, H_{0}(\widehat{d})\right\rangle+\left\langle\bar{\mu}_{I_{0}}, \widehat{v}\right\rangle<0
$$

Since $\left\langle\bar{\mu}_{I_{0}}, \widehat{v}\right\rangle \geqslant 0$, it follows from (25) that

$$
\left\langle\bar{\mu}_{I_{0}}, G_{0}^{I_{0}}(\widehat{d})\right\rangle+\left\langle\bar{\nu}, H_{0}(\widehat{d})\right\rangle<0
$$

which contradicts (22). Consequently, $\bar{\lambda}>0$, which completes the proof. 


\section{REFERENCES}

[1] F.H. Clarke, Optimization and nonsmooth analysis, Canadian Math. Soc. Series of Monographs and Advanced Texts (J. Wiley \& Sons, New York, 1983).

[2] B.D. Craven, 'Invex function and constrained local minima', Bull. Austral. Math. Soc. 24 (1981), 357-366.

[3] B.D. Craven, 'Relations between invex properties', World Sci. Ser. Appl. Anal. 5 (1995), 25-34.

[4] B.D. Craven, D.V. Luu and B.M. Glover, 'Strengthened invex and perturbations', Math. Methods Oper. Res. 43 (1996), 319-336.

[5] I.V. Girsanov, Lectures on mathematical theory of extremum problems (Springer-Verlag, Berlin, Heidelberg, 1972).

[6] M.A. Hanson, 'On sufficiency of the Kuhn-Tucker condition', J. Math. Anal. Appl. 80 (1981), 545-550.

[7] M.A. Hanson and N.G. Rueda, 'A sufficient condition for invexity', J. Math. Anal. Appl. 138 (1989), 193-198.

[8] N.X. Ha and D.V. Luu, 'Invexity of supremum and infimum functions', Bull. Austral. Math. Soc. 65 (2002), 289-306.

[9] A. Jourani, 'Constraint qualifications and Lagrange multipliers in nondifferentiable programming problems', J. Optim. Theory Appl. 81 (1994), 533-548.

[10] R.N. Kaul and S. Kaur, 'Optimality criteria in nonlinear programming involving nonconvex functions', J. Math. Anal. Appl. 105 (1985), 104-112.

[11] D.V. Luu and N.X. Ha, 'An invariant property of invex functions and applications', Acta Math. Vietnam. 25 (2000), 181-193.

[12] O.L. Mangasarian and S. Fromovitz, 'The Fritz-John necessary optimality conditions in the presence of equality and inequality constraints', J. Math. Anal. Appl. 17 (1967), $37-47$.

[13] V.H. Nguyen, J.J. Strodiot and R. Mifflin, 'On conditions to have bounded multipliers in locally Lipschitz programming', Math. Programming 18 (1980), 100-106.

[14] T.W. Reiland, 'Nonsmooth invexity', Bull. Austral. Math. Soc. 42 (1990), 437-446.

[15] R.T. Rockafellar, Convex analysis (Princeton University Press, Princeton, New Jersey, 1970).

[16] S.M. Robinson, 'Stability theory for systems of inequalities, Part II: Differentiable nonlinear systems', SIAM J. Numer. Anal. 13 (1976), 497-513.

Institute of Cryptographic Technology

Hanoi

Vietnam
Institute of Mathematics

PO Box 631 Bo Ho

10000 Hanoi

Vietnam 